\title{
Editorial
}

\section{RIGHT PROPOSALS}

As usual, we hope that this New Year becomes very productive for all professionals in our area and also in the correlated ones.

This issue includes 20 articles covering the fields of the Speech, Language and Hearing Sciences scope, especially Health and Education. The manuscripts, presented as original articles, review articles and case reports, were developed in several graduation and post graduation Institutions, from different regions of our country.

The timelines make us think and talk about "the first Journal of 2012." However, the studies presented in these manuscripts started a few years ago. They are the result of the will of researchers and clinicians to share their findings, based on the effort to find answers that frequently lead to future questions, creating the necessary movement for the development of our science.

It is a pleasure to share these experiences.

Searches for news - It is common to hear our students saying that certain subject does not matter because "we know much about it." They want to find something "very important" or "unprecedented" even though they can say, afterwards, they found nothing in the literature to make a good discussion...

Searching for deepening - how difficult it is to achieve a good systematic review of the literature. The researcher may come across different methodological approaches, conflicting or overlapping opinions. It is also difficult to get further from controlled studies - structure of the method, reproducibility, control of the variables, interpretation of data - not to mention the discussions about "objective and subjective data" (the belief that numbers bring undeniable results).

There were no "objective data" without interpretation, and that depends on the knowledge, experiences and focus of the researchers.

Searching for discoveries - how important it is to communicate what was observed in clinical practice, using data from trial participants or given case. That is the reason to report those special cases that trigger new interests or those that confirm known approaches.

Anyway, to get a final product that is to write with nimbleness, about what "you" know or came to know with scientific research.

And so we start over. I have the great pleasure of accompanying the Journal CEFAC as its scientific editor one year more. Many old challenges remain while new ones will always appear to test our ability to continue fighting. The battle continues, and especially the optimism too. Ever! After all, it is a proud to be a Speech and Language Therapist.

....and trying to keep up the speed of information in today's world!

Esther Mandelbaum Gonçalves Bianchini Scientific Editor

Speech, Language, Hearing Sciences and Education Journal 